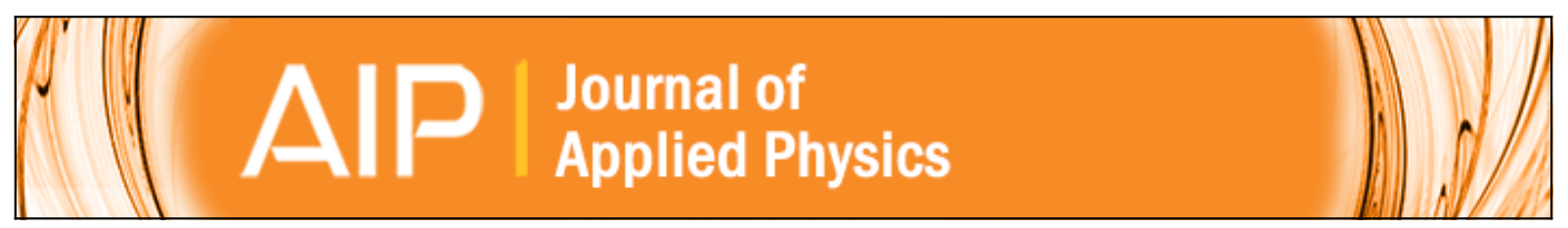

\title{
Robust superconductivity with large upper critical field in Nb2PdS5
}

Rajveer Jha, Brajesh Tiwari, Poonam Rani, Hari Kishan, and V. P. S. Awana

Citation: Journal of Applied Physics 115, 213903 (2014); doi: 10.1063/1.4881278

View online: http://dx.doi.org/10.1063/1.4881278

View Table of Contents: http://scitation.aip.org/content/aip/journal/jap/115/21 ?ver=pdfcov

Published by the AIP Publishing

\section{Articles you may be interested in}

Superconductivity in BiS 2 based $\mathrm{Bi} 4 \mathrm{O} 4 \mathrm{~S} 3$ novel compound

AIP Conf. Proc. 1512, 1104 (2013); 10.1063/1.4791432

Interstitial doping induced superconductivity at $15.3 \mathrm{~K}$ in Nb5Ge3 compound

J. Appl. Phys. 111, 123912 (2012); 10.1063/1.4730611

Angular dependence of pinning potential, upper critical field, and irreversibility field in underdoped BaFe1.9Co0.1As2 single crystal

Appl. Phys. Lett. 100, 102601 (2012); 10.1063/1.3692582

Superconductivity In Y2Pd(Ge1x Si x $) 3(x=01)$

AIP Conf. Proc. 850, 649 (2006); 10.1063/1.2354877

Superconducting Transition Temperature of (Nb1xZrx)0.8B2

AIP Conf. Proc. 850, 639 (2006); 10.1063/1.2354872

\section{AlP $\mid$ Applied Physics Letters}

is pleased to announce Reuben Collins as its new Editor-in-Chief

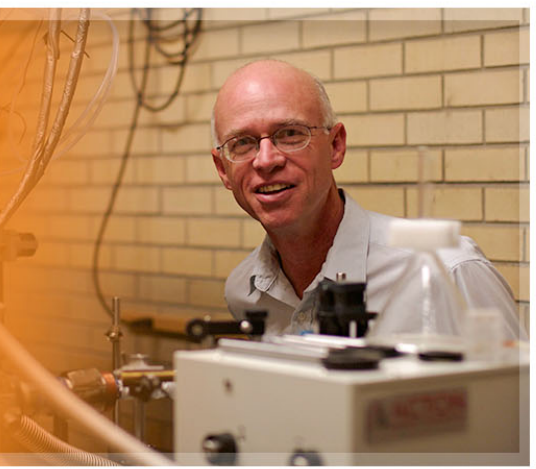




\title{
Robust superconductivity with large upper critical field in $\mathbf{N b}_{\mathbf{2}} \mathbf{P d S _ { 5 }}$
}

\author{
Rajveer Jha, Brajesh Tiwari, Poonam Rani, Hari Kishan, and V. P. S. Awana ${ }^{\text {a) }}$ \\ Quantum Phenomena and Applications Division, National Physical Laboratory (CSIR), \\ Dr. K. S. Krishnan Road, New Delhi-110012, India
}

(Received 25 April 2014; accepted 21 May 2014; published online 3 June 2014)

\begin{abstract}
We report synthesis, structural details, and complete superconducting characterization of very recently discovered [Q. Zhang, Sci. Rep. 3, 1446 (2013)] $\mathrm{Nb}_{2} \mathrm{PdS}_{5}$ new superconductor. The synthesized compound is crystallized in monoclinic structure with $C 2 / m$ (\#12) space group. Bulk superconductivity is seen in both $a c / d c$ magnetic susceptibility and electrical resistivity measurements with superconducting transition temperature $\left(T_{c}\right)$ at $6 \mathrm{~K}$. The upper critical field $\left(H_{c 2}\right)$ being estimated from high field magneto-transport $[\rho(T) H]$ measurements is above $240 \mathrm{kOe}$. The estimated $H_{c 2}(0)$ is clearly above the Pauli paramagnetic limit of $\sim 1.84 T_{c}$. Heat capacity $\left(C_{p}\right)$ measurements show clear transition with well defined peak at $T_{c}$, but with lower jump than as expected for a Bardeen-Cooper-Schrieffer type superconductor. The Sommerfeld constant $(\gamma)$ and Debye temperature $\left(\Theta_{D}\right)$ as determined from low temperature fitting of $C_{P}(T)$ data are $32 \mathrm{~mJ} / \mathrm{mole}-\mathrm{K}^{2}$ and $263 \mathrm{~K}$, respectively. Hall coefficients and resistivity in conjugation with electronic heat capacity indicates multiple gap superconductivity signatures in $\mathrm{Nb}_{2} \mathrm{PdS}_{5}$. We also studied the impact of hydrostatic pressure $(0-1.97 \mathrm{Gpa})$ on superconductivity of $\mathrm{Nb}_{2} \mathrm{PdS}_{5}$ and found nearly no change in $T_{c}$ for the given pressure range. (C) 2014 AIP Publishing LLC. [http://dx.doi.org/10.1063/1.4881278]
\end{abstract}

\section{INTRODUCTION}

Very recently discovered superconductivity in layered $\mathrm{Nb}_{2} \mathrm{PdS}_{5}$ along with superconductivity of Fe based oxypnictides i.e., REFeAsO/F has attracted huge attention of scientific community. ${ }^{1,2}$ In fact, seemingly it was like second revolution after the invention of high $\mathrm{T}_{\mathrm{c}}$ cuprates superconductivity way back in 1986 (Ref. 3) Though other superconductors like famous $\mathrm{MgB}_{2}$ keep on adding, ${ }^{4}$ but it is only after the Fe pnictides that search for new superconductors is sparked once again. Besides several Fe pnictide ${ }^{5,6}$ and chalcagonide $^{7,8}$ families, some other similar structure compounds viz. the $\mathrm{BiS}_{2}$ based $^{9-11}$ ones are added more recently. When looking for new superconductors, one aspires for best superconducting properties, such as higher critical temperature $\left(T_{c}\right)$ and upper critical field $\left(H_{c 2}\right)$. In general, it is a feast for a scientist to discover a higher $T_{c}$ and $H_{c 2}$ superconductor. Both parameters are important for practical applications of a superconductor. Besides the higher $T_{c}$, which helps in reducing the operating temperature cost, the upper critical field warrants the robustness of the superconductor against magnetic field. In this direction, the recently discovered $\mathrm{Nb}_{2} \mathrm{PdS}_{5}$ superconductor, ${ }^{1,12}$ though possess comparatively lower $T_{c}$ of around $6 \mathrm{~K}$ only, but the same is quite robust against magnetic field.

The robustness of superconductivity does mean here that superconductivity is least affected by the applied magnetic field. Generally speaking, the decrease of $T_{c}$ with applied magnetic field, i.e., $\mathrm{d} T_{c} / \mathrm{d} H$ determines the upper critical field in a simple single band scenario. ${ }^{13}$ In a type II superconductor, the upper critical field can be enhanced by both intrinsically $^{6,14}$ and extrinsically. ${ }^{15}$ Intrinsically, the same happens

\footnotetext{
a) Author to whom correspondence should be addressed. E-mail: awana@mail.npindia.org. Telephone +91-11-45609357, Fax: +91-1145609310
}

in multi band systems having complicated electronic ${ }^{6,14}$ and extrinsically by pinning the vortices in their mixed state. ${ }^{15}$ The recently discovered superconductor, i.e., $\mathrm{Nb}_{2} \mathrm{PdS}_{5}$ seems to be both intrinsically robust as well as pinned by inherent defects, etc. It is noteworthy here that $\mathrm{Nb}$ is the most popular superconductor being used till date in superconductor industry having varying superconducting transition temperature $\left(T_{c}\right)$ values in various metal or alloy forms from $9 \mathrm{~K}(\mathrm{Nb})$, $11.5 \mathrm{~K}(\mathrm{NbC}), 16 \mathrm{~K}(\mathrm{NbN}), 19 \mathrm{~K}\left(\mathrm{Nb}_{3} \mathrm{Sn}\right)$ to $23 \mathrm{~K}\left(\mathrm{Nb}_{3} \mathrm{Ge}\right) .{ }^{16-20}$

The recent compound, i.e., $\mathrm{Nb}_{2} \mathrm{PdS}_{5}$ somehow reminds not only the importance of $\mathrm{Nb}$ being the part of various superconductors in past, ${ }^{16-20}$ but also the presence of $\mathrm{S}$ in its layered structure calls for the attention of famous high upper critical field $\mathrm{S}$ containing Chevrel phase compounds. ${ }^{21}$ Keeping in view the importance of $\mathrm{Nb}$ and the utility of $\mathrm{S}$ in new layered structure of the recent compound $\mathrm{Nb}_{2} \mathrm{PdS}_{5}$, we present here an easy and clear route of synthesis of this new superconductor. Further full superconducting characterization in terms of high field (140 kOe) and low temperature (down to $2 \mathrm{~K}$ ), electrical, magnetic, and thermal characterizations are presented in current communication.

\section{EXPERIMENT}

Polycrystalline bulk $\mathrm{Nb}_{2} \mathrm{PdS}_{5}$ is synthesized via solid state reaction route by quartz vacuum encapsulation technique at $850^{\circ} \mathrm{C}$. The high purity $(4 \mathrm{~N})$ ingredients, i.e., $\mathrm{Nb}, \mathrm{Pd}$, and $\mathrm{S}$ are weighed in stoichiometric ratio and mixed thoroughly in a glove box in Ar atmosphere. The mixed powder is pelletized, sealed in an evacuated quartz tube, and put immediately in box furnace. The sample is heated to $850^{\circ} \mathrm{C}$ with heating rate of $3 \mathrm{~min}$ and hold at same temperature for $24 \mathrm{~h}$. Subsequently, the furnace is switched off and allowed to cool to room temperature over a span of $6 \mathrm{~h}$. Thus, obtained sample is once again pulverized, pelletized, sealed in encapsulated 


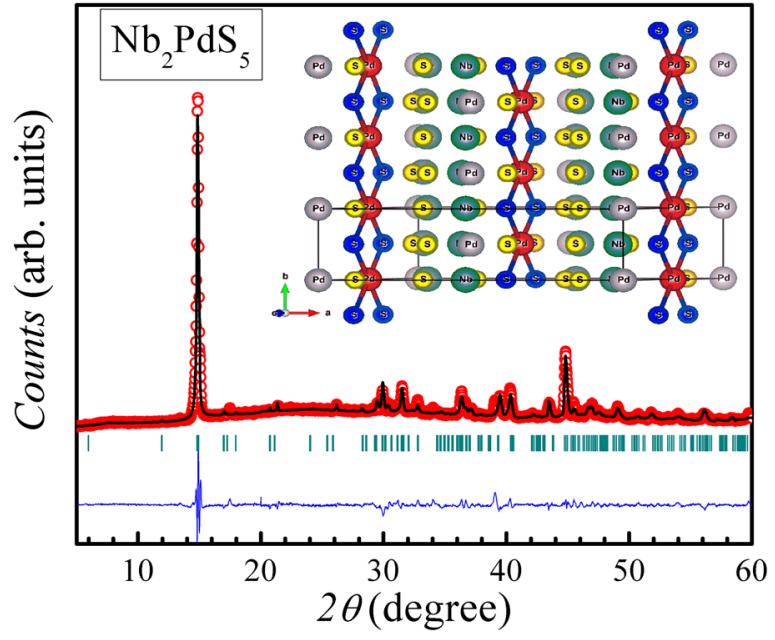

FIG. 1. Rietveld refined Room temperature $X R \mathrm{D}$ patterns of $\mathrm{Nb}_{2} \mathrm{PdS}_{5}$, the unit cell of the compound is shown in inset.

quartz tube, heated at same temperature for another $24 \mathrm{~h}$, and furnace cooled to room temperature. The room temperature $\mathrm{X}$-ray diffraction $(X R D)$ patterns are taken after each heat treatment on Rigaku XRD machine. The electrical, magnetic, and thermal characterization is done with help of Quantum Design ( $Q D)$ Physical Property Measurement System $(P P M S)-140 \mathrm{kOe}$ down to $2 \mathrm{~K}$. Hydrostatic pressures are generated by a $\mathrm{BeCu} / \mathrm{NiCrAl}$ clamped piston-cylinder cell using HPC-33, an addition to PPMS QD, to study the pressure effect on $\mathrm{Nb}_{2} \mathrm{PdS}_{5}$. The pressure at low temperature was calibrated from the superconducting transition temperature of $\mathrm{Pb}$. The sample is immersed in a fluid pressure transmitting medium of Fluorinert in a Teflon cell. Annealed Pt wires were affixed to gold-sputtered contact surfaces on each sample with silver epoxy in a standard four-wire configuration.

\section{RESULTS AND DISCUSSION}

The synthesized $\mathrm{Nb}_{2} \mathrm{PdS}_{5}$ sample is gray in color. The room temperature fitted and observed $X R D$ pattern for the synthesized $\mathrm{Nb}_{2} \mathrm{PdS}_{5}$ along with its unit cell is shown in Figure 1. $\mathrm{Nb}_{2} \mathrm{PdS}_{5}$ crystallizes in Centro-symmetric structure within space group $C 2 / m$ (\#12). There are two formula units in each unit cell comprising two sites each for $\mathrm{Nb}(\mathrm{Nb} 1$, $\mathrm{Nb} 2)$ and $\mathrm{Pd}(\mathrm{Pd} 1, \mathrm{Pd} 2)$ along with five $\mathrm{S}$ sites (S1, S2, S3, $\mathrm{S} 4, \mathrm{~S} 5)$. The schematic sketch of the unit cell is shown in inset of Fig. 1. Details of Wyckoff positions, site symmetry, and fractional occupancies being obtained from Rietveld fitting of the experimental XRD data are given in Table I. The lattice parameters are $a=12.134(2) \AA, \quad b=3.277(1) \AA$, $c=15.023(3) \AA$, with $\alpha=90^{\circ}, \beta=103.23^{\circ}(1)$ and $\gamma=90^{\circ}$. Structural detail results are in general agreement with another recently discovered similar superconducting compound $\mathrm{Ta}_{2} \mathrm{PdS}_{5}{ }^{22}$

In a very recent electronic structure determination theoretical article, ${ }^{23}$ slightly different configuration is used, i.e., high conductivity direction is labeled along $c$-axis instead of usual $b$-axis. ${ }^{1,22}$ In fact due to anisotropic nature of the conductivity in different $\mathrm{Pd}-\mathrm{S}$ chains/sheets, the superconducting upper critical field is highly directional dependent in
TABLE I. Rietveld refined Wyckoff positions and fractional occupancies of the atoms in $\mathrm{Nb}_{2} \mathrm{PdS}_{5}$.

\begin{tabular}{llclcc}
\hline \hline Atom & \multicolumn{1}{c}{$\mathrm{x}$} & $\mathrm{y}$ & \multicolumn{1}{c}{$\mathrm{z}$} & site & Fractional occupancy \\
\hline $\mathrm{Nb} 1$ & $0.0759(5)$ & 0.5000 & $0.179(4)$ & $4 \mathrm{i}$ & 1 \\
$\mathrm{Nb} 2$ & $0.1528(8)$ & 0.0000 & $0.3783(2)$ & $4 \mathrm{i}$ & 1 \\
$\mathrm{Pd} 1$ & 0.0000 & 0.0000 & 0.0000 & $2 \mathrm{a}$ & $1 / 2$ \\
$\mathrm{Pd} 2$ & 0.0000 & 0.0000 & $0.5000)$ & $2 \mathrm{c}$ & $1 / 2$ \\
$\mathrm{~S} 1$ & $0.3503(6)$ & 0.0000 & $0.4890(1)$ & $4 \mathrm{i}$ & 1 \\
$\mathrm{~S} 2$ & $0.2537(4)$ & 0.5000 & $0.2950(6)$ & $4 \mathrm{i}$ & 1 \\
$\mathrm{~S} 3$ & $0.1753(6)$ & 0.0000 & $0.0977(8))$ & $4 \mathrm{i}$ & 1 \\
$\mathrm{~S} 4$ & $0.4230(3)$ & 0.5000 & $0.1321(4)$ & $4 \mathrm{i}$ & 1 \\
$\mathrm{~S} 5$ & $0.4904(7)$ & 0.0000 & $0.3234(6)$ & $4 \mathrm{i}$ & 1 \\
\hline \hline
\end{tabular}

these compounds. ${ }^{1,22,23}$ In a more recent communication, ${ }^{24}$ the $\mathrm{Nb}_{2} \mathrm{Pd}_{\mathrm{x}} \mathrm{S}_{5-\mathrm{y}}$ fiber exhibited slightly modified superconducting parameters including higher $T_{c}$ of $7.14 \mathrm{~K}$. The role of off stoichiometry and directional alignment could lead to changed superconducting characteristics. Here, we stick to stoichiometric $\mathrm{Nb}_{2} \mathrm{PdS}_{5}$ bulk polycrystalline compound to minimize the complications.

$A C$ susceptibility versus temperature $\chi(T)$ behavior of the $\mathrm{Nb}_{2} \mathrm{PdS}_{5}$ sample is depicted in Figure 2. AC susceptibility measurements are done at $33 \mathrm{~Hz}$ and $10 \mathrm{Oe} A C$ drive field. $D C$ applied field is kept zero to check the superconducting transition temperature $\left(T_{c}\right)$. Both the real $\left(\chi^{\prime}\right)$ and imaginary $\left(\chi^{\prime \prime}\right)$ part of $a c$ susceptibility are measured. Real part $\left(\chi^{\prime}\right)$ susceptibility shows transition to diamagnetism at around $5.8 \mathrm{~K}$, confirming bulk superconductivity. The imaginary part on the other hand exhibits a single sharp peak in positive susceptibility at around the same temperature. Presence of single sharp peak in $\chi^{\prime \prime}$ is reminiscent of better superconducting grains coupling in studied $\mathrm{Nb}_{2} \mathrm{PdS}_{5}$ superconductor. We also measured the $A C$ susceptibility of the compound in applied $d c$ fields of up to $120 \mathrm{kOe}$. The plot of real part $\left(\chi^{\prime}\right)$ of $a c$ susceptibility with applied field is depicted in inset of Figure 2. The temperature is fixed at $3 \mathrm{~K}$, i.e., well below the superconducting transition temperature

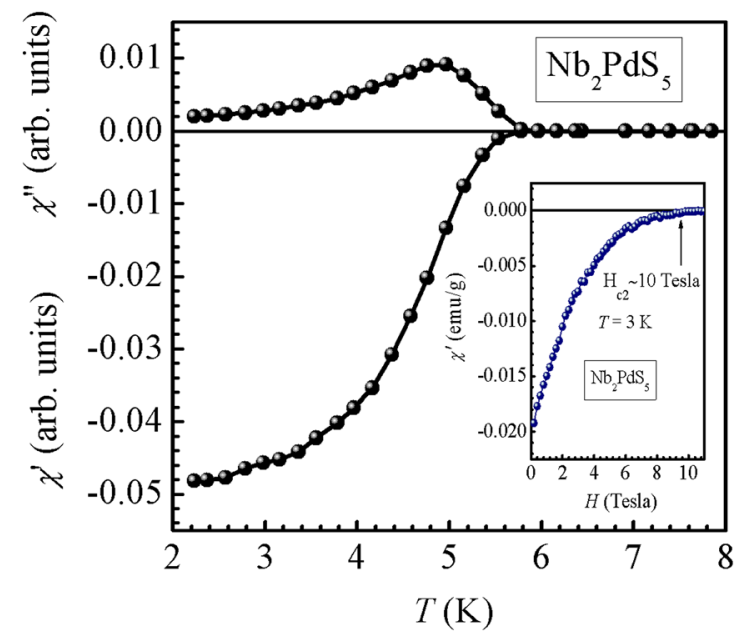

FIG. 2. $A C$ susceptibility $\chi(T)$ behavior of the $\mathrm{Nb}_{2} \mathrm{PdS}_{5}$ sample at frequency $333 \mathrm{~Hz}$ and $a c$ drive amplitude of $10 \mathrm{Oe}$, the inset shows the real part of the same in applied dc fields of up to $120 \mathrm{kOe}$. 


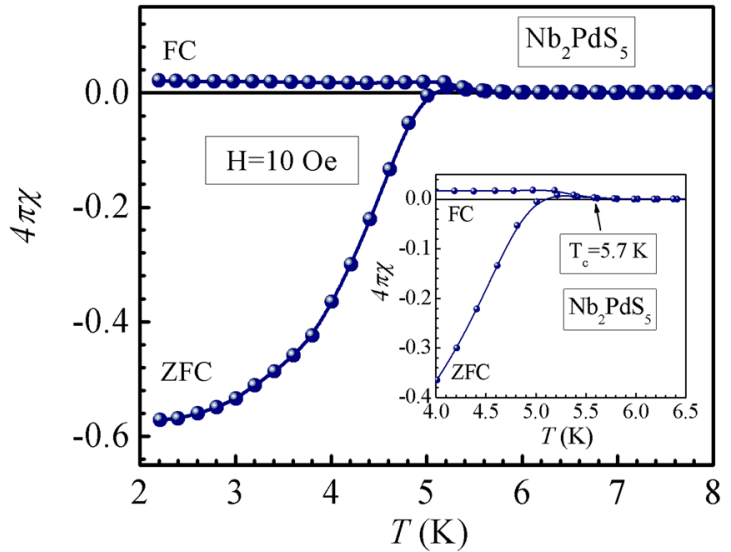

FIG. 3. Temperature variation of $D C$ Magnetization in $Z F C$ and $F C$ modes for $\mathrm{Nb}_{2} \mathrm{PdS}_{5}$ compound at $10 \mathrm{Oe}$, inset shows the expanded part of the same plot indicating irreversible behavior and marking of $T_{c}$.

of $6 \mathrm{~K}$. It is clear from inset of Fig. 2 that the studied $\mathrm{Nb}_{2} \mathrm{PdS}_{5}$ is bulk superconducting (diamagnetic) at $3 \mathrm{~K}$ in up to $100 \mathrm{kOe}$ applied field. The upper critical field $\left(\mathrm{Hc}_{2}\right)$ is above $100 \mathrm{kOe}$ just $3 \mathrm{~K}$ below the superconducting transition temperature $\left(T_{c}\right)$. This is first indication that $\mathrm{Nb}_{2} \mathrm{PdS}_{5}$ is quite robust superconductor against magnetic field. More will be discussed after magneto-transport results in the following paragraphs.

DC magnetic susceptibility of $\mathrm{Nb}_{2} \mathrm{PdS}_{5}$ sample is shown in Figure 3. The magnetization is done in both $F C$ (Field cooled) and ZFC (Zero-field-cooled) protocol under applied magnetic field of $10 \mathrm{Oe}$. The compound shows superconducting onset, in terms of FC and ZFC bifurcation from $5.7 \mathrm{~K}$. This is clear from the zoomed inset of Figure 3. There is evidence for substantial flux trapping too. The bifurcation of $F C$ and $Z F C$ below $T_{c}$ marks the irreversible region. The shielding fraction as evidenced from $Z F C$ diamagnetic susceptibility is quite appreciable $(\sim 58 \%)$. An interesting fact we found repeatedly in case of $\mathrm{Nb}_{2} \mathrm{PdS}_{5}$ superconductor is the appearance of paramagnetic meissner effect $(P M E)$ in $F C$ magnetization. $P M E$ generally appears in heavily pinned superconductors $^{25}$ and could be a good prior indication of high $\mathrm{Hc}_{2}$.

Figure 4 depicts the resistivity versus temperature $(\rho-T)$ measurement with and without applied magnetic field. The resistance of the sample decreases with temperature and confirms superconductivity with onset $T_{c} \sim 6.3 \mathrm{~K}$ and $T_{c}(\rho=0)$ at $5.9 \mathrm{~K}$. The normal state conduction is of metallic type. From the fitting of resistivity $\rho=\rho_{\mathrm{o}}+\mathrm{AT}^{2}$ for low temperatures $\left(\mathrm{T}_{\mathrm{c}}<\mathrm{T}<50 \mathrm{~K}\right)$, we obtained residual resistivity $\rho_{\mathrm{o}}=1.27 \mathrm{~m} \Omega-\mathrm{cm}$ and $\mathrm{A}=1.126 \times 10^{-4} \mathrm{~m} \Omega-\mathrm{cm} / \mathrm{K}^{2}$ values. The quadratic variation in resistivity with temperature is shown as red solid curve in Fig. 4, which is a clear indication of Fermi-liquid nature of $\mathrm{Nb}_{2} \mathrm{PdS}_{5}$. To study the impact of applied magnetic field on superconductivity of $\mathrm{Nb}_{2} \mathrm{PdS}_{5}$ compound, the magneto-transport measurements are carried out in superconducting region, and the results are shown in inset-I of Fig. 4. With applied field of $110 \mathrm{kOe}$, the $T_{c}$ $(\rho=0)$ decreases from $5.9 \mathrm{~K}$ (zero-field) to $2.4 \mathrm{~K}(110 \mathrm{KOe})$. As sketched in inset-II of Fig. 4, we have estimated upper critical field $H_{c 2}(T)$ by using the conservative procedure of

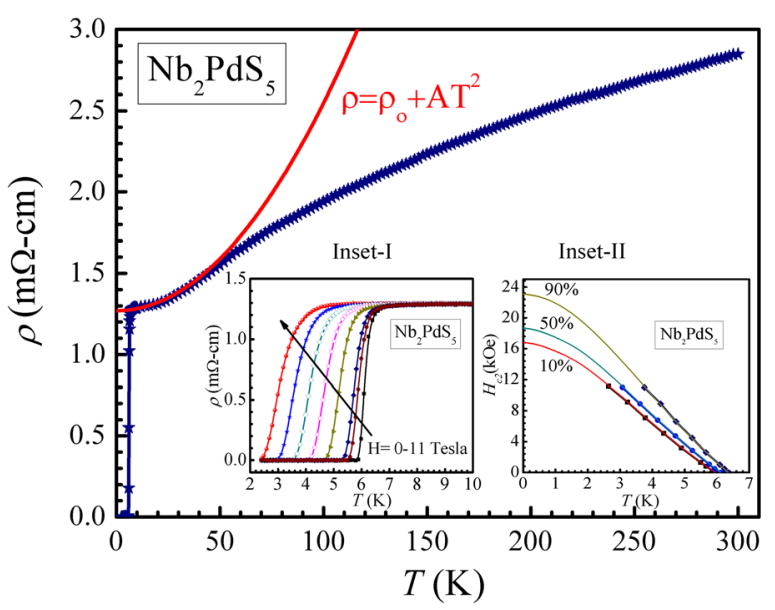

FIG. 4. Resistivity vs. temperature $(\rho-T)$ behavior of $\mathrm{Nb}_{2} \mathrm{PdS}_{5}$, inset-I shows the same in various applied fields of $0-110 \mathrm{kOe}$ below $10 \mathrm{~K}$ and inset-II is the upper critical field estimated from the $\rho(T) H$ data with $90 \%, 50 \%$, and $10 \% \rho_{n}$ criteria.

$90 \%, 50 \%$, and $10 \%$ dropping of resistivity from normal state into the superconducting transition line. While the applicability of WHH (Werthamer-Helfand-Hohenberg) approximation can be debated in this new superconductor, a simplistic single band extrapolation leads to $H_{c 2}(0)(=-0.69$ $\mathrm{T}_{\mathrm{c}} \mathrm{d} H_{c 2} /\left.\mathrm{d} T\right|_{T c}$ ) value of $240 \mathrm{kOe}, 185 \mathrm{kOe}$, and $170 \mathrm{kOe}$ with of $90 \%, 50 \%$, and $10 \%$ criterion, respectively. These values match with the experimentally ascertained bulk diamagnetic response of $\mathrm{Nb}_{2} \mathrm{PdS}_{5}$ superconductor at $3 \mathrm{~K}$ in $100 \mathrm{kOe}$ field (inset Fig. 2). The estimated values of upper critical field are close to the earlier reported values. ${ }^{12}$ Interestingly, the $H_{c 2}(0)$ value for $\mathrm{Nb}_{2} \mathrm{PdS}_{5}$ superconductor is more than the one as being expected within Pauli Paramagnetic limit of $H_{c 2}(0)=1.84 T_{c}$ of around $110 \mathrm{kOe}$.

The relative decrement of superconductivity with applied field i.e., $\mathrm{d} T_{c} / \mathrm{d} H$ is around $0.3 \mathrm{~K} / 10 \mathrm{kOe}$ from absolute $T_{c}$ $(\rho=0)$ criteria. When this is compared with the best of HTSc cuprates $(3 \mathrm{~K} / 10 \mathrm{kOe}),{ }^{26} \mathrm{Fe}$ pnictides $(1 \mathrm{~K} / 10 \mathrm{kOe}),{ }^{6,14}$ pinned $\mathrm{MgB}_{2}(1.5 \mathrm{~K} / 10 \mathrm{kOe}),{ }^{15}$ or the $\mathrm{Fe}$ chalcegonides $(0.4 \mathrm{~K} / 10 \mathrm{kOe})^{7,8}$ and Chevrel phase $(0.45 \mathrm{~K} / 10 \mathrm{kOe})^{27} \mathrm{com}-$ pounds, one finds that $\mathrm{Nb}_{2} \mathrm{PdS}_{5}$ is the most robust superconductor against magnetic field till date.

Figure 5 shows the heat capacity $\left(C_{P}\right)$ versus temperature $(T)$ plots at different applied fields of $0,10,50$, and $140 \mathrm{kOe}$ in superconducting region i.e., $2-8 \mathrm{~K}$ for studied $\mathrm{Nb}_{2} \mathrm{PdS}_{5}$ superconductor. In normal state i.e., at $200 \mathrm{~K}$, the value of $C_{P}$ is around $180 \mathrm{~J} / \mathrm{mole} \mathrm{K}$, see inset of Figure 5 . The superconducting transition temperature $\left(T_{c}\right)$ is seen clearly as a hump in $C_{p}$ at around $6 \mathrm{~K}$. As evident from Fig. 5 , the $C_{p}$ peak temperature shifts to lower temperatures with application of external field and is namely at $5.5 \mathrm{~K}, 5.2 \mathrm{~K}$, and $4.2 \mathrm{~K}$ at $0,10 \mathrm{kOe}$, and $50 \mathrm{kOe}$, respectively. The $C_{p}$ hump and associated peak temperature is not seen down to $2 \mathrm{~K}$ at $140 \mathrm{kOe}$ magnetic field. This shows the compound is not superconducting down to $2 \mathrm{~K}$ in applied field of $140 \mathrm{kOe}$.

Figure 6 shows the low temperature normal-state $140 \mathrm{KOe} C_{p}(T)$ data from $2-10 \mathrm{~K}$, which is fitted to the Sommerfeld-Debye expression as $C_{p}(T)=\gamma T+\beta T^{3}+\delta T^{5}$. The values of Sommerfeld constant $(\gamma)$ and $\beta$ are obtained 


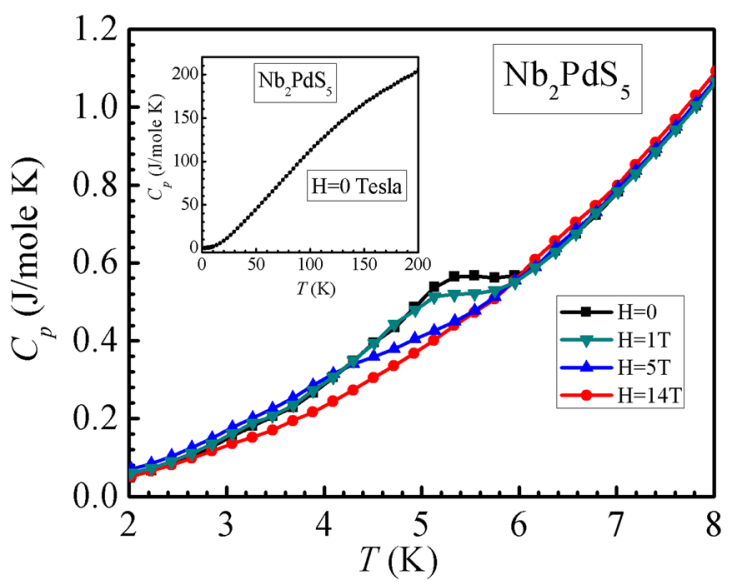

FIG. 5. Heat capacity $\left(C_{p}\right)$ versus temperature plot for $\mathrm{Nb}_{2} \mathrm{PdS}_{5}$ superconductor; inset shows the same in $0,10,50$, and $140 \mathrm{kOe}$ fields in superconductivity region below $8 \mathrm{~K}$.

from the fitting of the experimental data are as $\gamma=32 \mathrm{~mJ} / \mathrm{mol}^{-\mathrm{K}^{2}}, \quad \beta=1.79 \mathrm{~mJ} / \mathrm{mol}^{-} \mathrm{K}^{4}$ and $\delta=-3.48$ $\times 10^{-3} \mathrm{~mJ} / \mathrm{mol} \mathrm{K}^{6}$. The heat capacity jump $\left(\Delta C=C_{s^{-}} C_{p}\right)$ is estimated by subtracting superconducting heat capacity $\left(C_{s}\right)$ to the normal state fitted $C_{p}$ and the same is shown in the inset of Fig. 6. The normalized value of jump $\left(\Delta C / \gamma T_{c}\right)$ is $\sim 0.75$, which is less than in comparison to the Bardeen-Cooper-Schrieffer $(B C S)$ value of 1.43. As seen from inset of Fig. 6, the $C_{p}$ peak exhibits multiple gap superconductivity signatures with three different $T_{c}$, with slight upturn near $2 \mathrm{~K}$. The relatively higher value of $\gamma=35 \mathrm{~mJ} / \mathrm{mol}-\mathrm{K}^{2}$ is an indication that the $\mathrm{Nb}_{2} \mathrm{PdS}_{5}$ is a strongly coupled superconductor. ${ }^{23}$ Worth mentioning is the fact that $\beta$ and $\gamma$ values change slightly, when instead of extended low $T$ fitting of experimental $C_{p}$ to normal state, the $140 \mathrm{kOe}$ low $T$ experimental data are taken as normal state. Yet, the fact remains that the normalized value of $C_{p}$ jump is smaller than $B C S$ value and the same clearly demonstrates the multi gap superconductivity. The Debye temperature is calculated by using $\Theta_{\mathrm{D}}=(234 z R / \beta)^{1 / 3}$; here, $z$ being number of atoms per formula unit and $R$ is the gas constant. Taking the fitted value of $\beta\left(1.79 \mathrm{~mJ} / \mathrm{mol}^{-} \mathrm{K}^{4}\right)$, the calculated value of $\Theta_{D}$ is $263 \mathrm{~K}$.

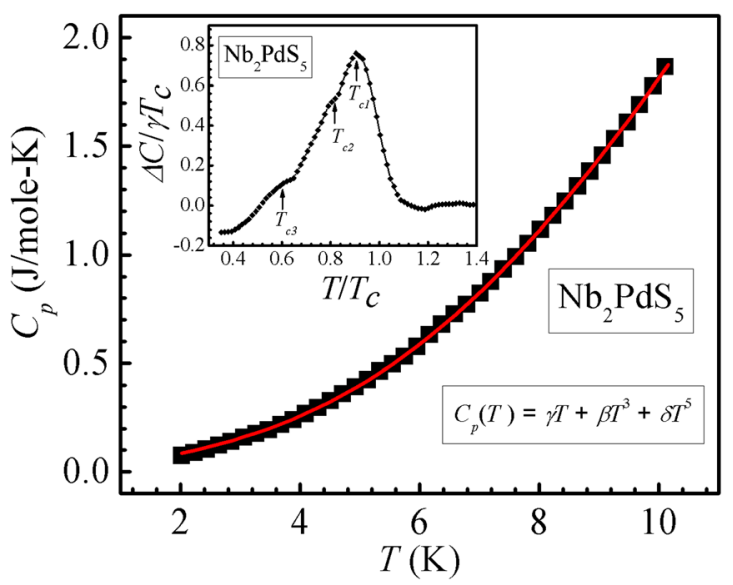

FIG. 6. Fitted (red line) and observed (filled squares) $C p$ versus $T$ data for $\mathrm{Nb}_{2} \mathrm{PdS}_{5}$ at applied field of $140 \mathrm{kOe}$ in low temperature region, the inset shows the electronic specific heat peak at $T_{\mathrm{c}}$.

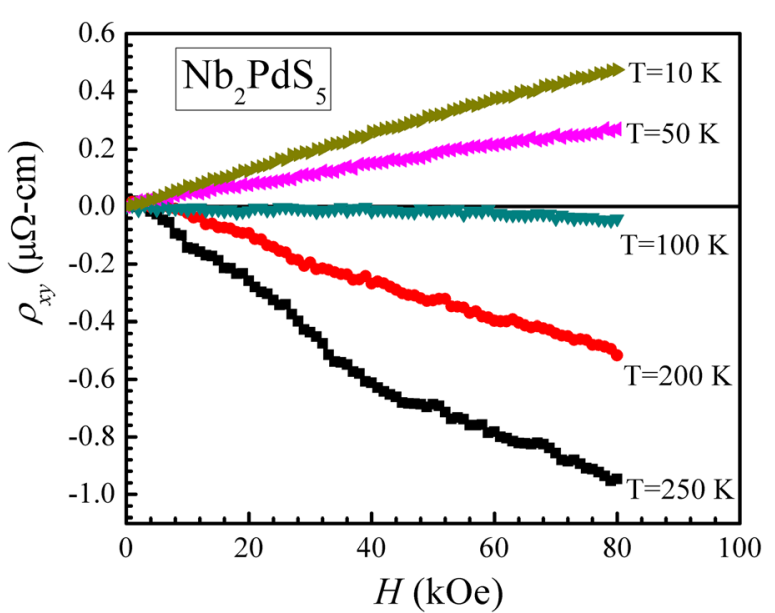

FIG. 7. Hall resistivity $\left(\rho_{x y}\right)$ versus magnetic field plots at various temperatures of $250 \mathrm{~K}, 200 \mathrm{~K}, 100 \mathrm{~K}, 50 \mathrm{~K}$, and $10 \mathrm{~K}$ for $\mathrm{Nb}_{2} \mathrm{PdS}_{5}$ superconductor.

In order to understand the carries contribution to transport properties in un-doped $\mathrm{Nb}_{2} \mathrm{PdS}_{5}$, Hall resistivity $\left(\rho_{x y}\right)$ and Hall coefficient $\left(R_{H}\right)$ measurements were carried out in normal state at different temperatures. Figure 7 depicts the Hall resistivity $\left(\rho_{x y}\right)$ results for studied $\mathrm{Nb}_{2} \mathrm{PdS}_{5}$ superconductor at various temperatures of $250 \mathrm{~K}, 200 \mathrm{~K}, 100 \mathrm{~K}, 50 \mathrm{~K}$, and $10 \mathrm{~K}$ up to applied magnetic field of $80 \mathrm{kOe}$. The magnetic field is applied perpendicular to the current flow direction and the voltage is recorded by five probe resistivity measurement method to minimize the magneto resistance contribution. It is clear from Fig. 7 that holes dominate the transport below temperatures $100 \mathrm{~K}$, whereas electrons at and above $100 \mathrm{~K}$. Clearly, this indicates that the $\mathrm{Nb}_{2} \mathrm{PdS}_{5}$ exhibits a change in type of dominating charge carriers at around $100 \mathrm{~K}$. To assert this behavior further, the Hall coefficient $\left(R_{H}\right)$ of $\mathrm{Nb}_{2} \mathrm{PdS}_{5}$ sample is measured under three different applied magnetic fields $5 \mathrm{kOe}, 30 \mathrm{kOe}$, and $50 \mathrm{kOe}$ in the temperature range of 2 to $300 \mathrm{~K}$, which is shown in Fig. 8. It can be observed from Fig. 8 that the $R_{H}$ changes its sign from negative (electron type) to positive (hole type) around $190 \mathrm{~K}$

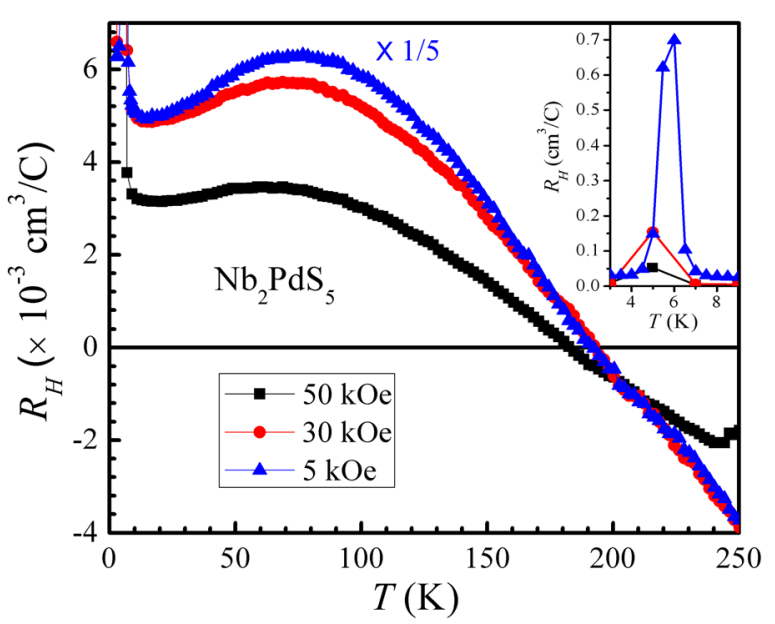

FIG. 8. Hall coefficient $\left(R_{H}\right)$ versus temperature plot for $\mathrm{Nb}_{2} \mathrm{PdS}_{5}$ superconductor under applied fields of $5 \mathrm{kOe}, 30 \mathrm{kOe}$, and $50 \mathrm{kOe} .5 \mathrm{kOe} \mathrm{R}_{\mathrm{H}}(T)$ is multiplied by $1 / 5$ for the clarity of viewgraph. Magnified graph close to superconducting transition is shown in inset. 


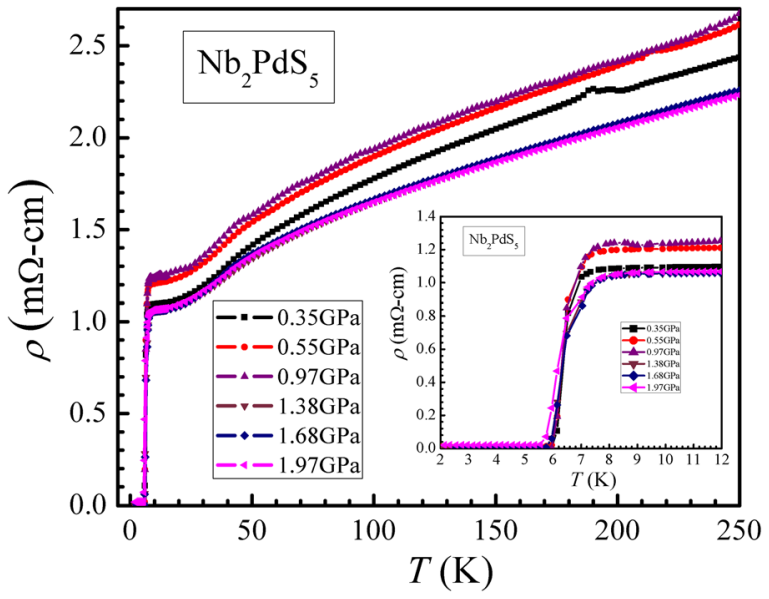

FIG. 9. Resistivity under different hydrostatic pressure $(0.35-1.98 \mathrm{GPa})$ as function of temperature for $\mathrm{Nb}_{2} \mathrm{PdS}_{5}$ superconductor, inset shows the magnified view of the same close to superconducting transition.

for all the three fields just after meeting together. $R_{H}$ is known to be sensitive to curvature of Fermi surface and renormalized Fermi velocity which may lead to change in the sign of Hall coefficient with temperature. ${ }^{28}$ Alternatively, this behavior can be understood by compensated two band model as proposed by Rullier-Albenque et al. to explain transport behavior of LiFeAs. ${ }^{29}$ This compensated two band transport property in normal state corroborates multiband superconductivity as claimed by heat capacity study for $\mathrm{Nb}_{2} \mathrm{PdS}_{5}$ compound. Spectroscopic investigations need to further furnish this claim. It is also observed that in normal state, the maximum of $R_{H}$ (i.e., carrier concentration, $\mathrm{n}$, is minimum) is at $T_{R H}(\max ) \sim 80 \mathrm{~K}$ even for three different magnetic fields though value of $R_{H}$ differs. It is interesting to note that this temperature $\left[T_{R H}(\max )\right]$ is very close to temperature at which Hall resistivity $\left(\rho_{x y}\right)$ changes its sign from negative to positive and not at which $R_{H}$ changes its sign. Hall coefficient $\left(R_{H}\right)$ sharply peaked to maximum at superconducting transition as shown in the inset of Fig. 8. For the applied field of $5 \mathrm{kOe}, R_{H}=7.01 \mathrm{~cm}^{3} / \mathrm{C}$ which is equivalent to hole concentration $\mathrm{n}=1 / \mathrm{e} \cdot R_{H}=8.92 \times 10^{21} / \mathrm{cm}^{3}$. Similar type of carrier sign change is seen in another related superconductor $2 \mathrm{H}-\mathrm{NbS}_{2}{ }^{28,30}$ Interestingly, $2 \mathrm{H}-\mathrm{NbS}_{2}$ is a known superconductor with $T_{c}$ above $6 \mathrm{~K} \cdot{ }^{28,30,31}$ This prompted us to check if the reported superconductivity of $\mathrm{Nb}_{2} \mathrm{PdS}_{5}$ could arise possibly due to minor $2 \mathrm{H}-\mathrm{NbS}_{2}$ phase. To exclude the possibility of $2 \mathrm{H}-\mathrm{NbS}_{2}$ impurity phase driven superconductivity in $\mathrm{Nb}_{2} \mathrm{PdS}_{5}$, we synthesized the $\mathrm{NbS}_{2}$ compound by same heat treatment of quartz vacuum encapsulation and heating of up to $850{ }^{\circ} \mathrm{C}$. Thus obtained $\mathrm{NbS}_{2}$ is crystallized in $R 3 m$ space group with $a$ and $c$ parameters of $3.335 \AA$ and $17.86 \AA$ (Ref. 32 ) and is not superconducting down to $2 \mathrm{~K}$, plots not shown. This clearly excluded the possibility of appearance of $\mathrm{NbS}_{2}$ impurity driven superconductivity in $\mathrm{Nb}_{2} \mathrm{PdS}_{5}$. To further the investigation of transport properties of $\mathrm{Nb}_{2} \mathrm{PdS}_{5}$ resistivity as a function of temperature, $\rho(T)$, is measured under various hydrostatic pressures up to $1.98 \mathrm{GPa}$, which is shown in Fig. 9. There is no appreciable change on $T_{c}$ as can be seen from the inset of Fig. 9. The normal state resistivity is though slightly increased for lower pressures of up to $0.97 \mathrm{GPa}$, the same exhibits decreasing trend for 1.68 and $1.97 \mathrm{GPa}$ pressures. On the other hand, we found nearly no change in $T_{c}$.

\section{CONCLUSIONS}

In conclusion, we have synthesized the new layered sulfide $\mathrm{Nb}_{2} \mathrm{PdS}_{5}$ superconductor and established its bulk superconductivity by magnetization, transport, and heat capacity measurements below $6 \mathrm{~K}$, with high upper critical field outside Pauli paramagnetic limit. The $C_{p}$ measurements exhibited multiple gap superconductivity signatures with three different $T_{c}$. The Sommerfeld constant $(\gamma)$ and Debye temperature $\left(\Theta_{D}\right)$ as determined from low temperature fitting of $C_{P}(T)$ are $35 \mathrm{~mJ} / \mathrm{mole}-\mathrm{K}^{2}$ and $253 \mathrm{~K}$, respectively. Hall studies in conjugation to heat capacity measurements affirm the possibility of multiband superconductivity in $\mathrm{Nb}_{2} \mathrm{PdS}_{5}$.

\section{ACKNOWLEDGMENTS}

Authors would like to thank their Director Professor R. C. Budhani for his keen interest in the present work. This work was supported by DAE-SRC outstanding investigator award scheme to work on search for new superconductors. H. Kishan thanks CSIR for providing Emeritus Scientist Fellowship.

${ }^{1}$ Q. Zhang, G. Li, D. Rhodes, A. Kiswandhi, T. Besara, B. Zeng, J. Sun, T. Siegrist, M. D. Johannes, and L. Balicas, Sci. Rep. 3, 1446 (2013).

${ }^{2}$ Y. Kamihara, T. Watanabe, M. Hirano, and H. Hosono, J. Am. Chem. Soc. 130, 3296 (2008).

${ }^{3}$ J. G. Bednorz and K. A. Muller, Z. Phys. B 64, 189 (1986).

${ }^{4}$ J. Nagamatsu, N. Nakagawa, T. Muranaka, Y. Zenitani, and J. Akimitsu, Nature (London) 410, 63 (2001).

${ }^{5}$ X. H. Chen, T. Wu, G. Wu, R. H. Liu, H. Chen, and D. F. Fang, Nature (London) 453, 761 (2008).

${ }^{6}$ V. P. S. Awana, R. S. Meena, A. Pal, A. Vajpayee, K. V. R. Rao, and H. Kishan, Euro Phys. J B 79, 139 (2011).

${ }^{7}$ F. C. Hsu, J. Y. Luo, T. K. Chen, T. W. Huang, Y. C. Lee, Y. L. Huang, Y. Y. Chu, D. C. Yan, and M. K. Wu, Proc. Natl. Acad. Sci. U.S.A. 105, 14262 (2008).

${ }^{8}$ Y. Mizuguchi, F. Tomioka, S. Tsuda, T. Yamaguchi, and Y. Takano, Appl. Phys. Lett. 94, 012503 (2009).

${ }^{9}$ Y. Mizuguchi, S. Demura, K. Deguchi, Y. Takano, H. Fujihisa, Y. Gotoh, H. Izawa, and O. J. Miura, Phys. Soc. Jpn. 81, 114725 (2012).

${ }^{10}$ V. P. S. Awana, A. Kumar, R. Jha, S. K. Singh, A. Pal, Shruti, and S. P. Patnaik, Solid State Commun. 157, 21 (2013).

${ }^{11}$ R. Jha, A. Kumar, S. K. Singh, and V. P. S. Awana, J. Appl. Phys. 113, 056102 (2013).

${ }^{12}$ C. Q. Niu, J. H. Yang, Y. K. Li, B. Chen, N. Zhou, J.Chen, J. L. Jiang, B. Chen, X. X. Yang, C. Cao, J. Dai, and X. Xu, Phys. Rev. B 88, 104507 (2013).

${ }^{13}$ E. Helfand and N. R. Werthamer, Phys. Rev. 147, 288 (1966).

${ }^{14}$ A. Srivastava, A. Pal, S. Singh, C. Shekhar, H. K. Singh, V. P. S. Awana, and O. N. Srivastava, AIP Adv. 3, 092113 (2013).

${ }^{15}$ A. Vajpayee, R. Jha, A. K. Srivastava, H. Kishan, M. Tropeano, C. Ferdeghini, and V. P. S. Awana, Sup. Sci. Tech. 24, 045013 (2011).

${ }^{16}$ B. T. Matthias, T. Geballe, and V. Compton, Rev. Mod. Phys. 35, 1 (1963).

${ }^{17}$ M. Wells, M. Pickus, K. Kennedy, and V. Zackay, Phys. Rev. Lett. 12, 536 (1964).

${ }^{18}$ T. H. Courtney, J. Reintjes, and J. Wulff, J. Appl. Phys. 36, 660 (1965).

${ }^{19}$ B. T. Matthias, T. H. Geballe, S. Geller, and E. Corenzwit, Phys. Rev. B 95, 1435 (1954).

${ }^{20}$ G. Oya and E. J. Saur, J. Low Temp. Phys. 34, 569 (1979).

${ }^{21}$ B. Chevrel, M. Sergent, and J. J. Prigent, Solid State Chem. 3, 515 (1971).

${ }^{22}$ Y. F. Lu, T. Takayama, A. F. Bangura, Y. Katsura, D. Hashizume, and H. Takagi, J. Phys. Soc. Japan. 83, 023702 (2014). 
${ }^{23}$ D. J. Singh, Phys. Rev. B 88, 174508 (2013).

${ }^{24}$ H. Yu, M. Zuo, L. Zhang, S. Tan, C. Zhang, and Y. Zhang, J. Am. Chem. Soc. 135, 12987 (2013).

${ }^{25}$ W. Braunisch, N. Knauf, V. Kataev, S. Newhausen, R. Grutz, B. Roden, D. Khomskii, and D. Wohlleben, Phys. Rev. Lett. 68, 1908 (1992).

${ }^{26}$ N. P. Liyanawaduge, S. K. W. Singh, A. Kumar, R. Jha, B. S. B. Karunaratne, and V. P. S. Awana, Sup. Sci. Tech. 25, 035017 (2012).

${ }^{27}$ K. Okuda, M. Kitagawa, T. Sakakibara, and M. J. Date, Phys. Soc. Jpn. 48, 2157 (1980).
${ }^{28}$ D. V. Evtushinsky, A. A. Kordyuk, V. B. Zabolotnyy, D. S. Inosov, B. Büchner, H. Berger, L. Patthey, R. Follath, and S. V. Borisenko, Phys. Rev. Lett. 100, 236402 (2008).

${ }^{29}$ F. Rullier-Albenque, D. Colson, A. Forget, and H. Alloul, Phys. Rev. Lett. 109, 187005 (2012)

${ }^{30}$ I. Guillamón, H. Suderow, S. Vieira, L. Cario, P. Diener, and P. Rodière, Phys. Rev. Lett. 101, 166407 (2008).

${ }^{31}$ J. Kacmarcik, Z. Pribulova, C. Marcenat, T. Klein, P. Rodiere, L. Cario, and P. Samuely, Phys. Rev. B 82, 014518 (2010).

${ }^{32}$ B. Morosin, Acta Cryst. B 30, 551 (1974). 